\title{
Saúde e reclusão: de uma breve contextualização do fenómeno ao papel dos profissionais de saúde
}

Health and imprisonment: from a brief contextualization of the phenomenon to the role of health professionals

Santé et emprisonnement : d'une brève contextualisation du phénomène au rôle des professionnels de santé

\section{Gloria Jólluskin, Andreia de Castro-Rodrigues, Isabel Silva e Verónica} Fernandes

\section{(2) OpenEdition}

\section{Journals}

Edição electrónica

URL: http://journals.openedition.org/configuracoes/2430

DOI: $10.4000 /$ configuracoes. 2430

ISSN: 2182-7419

\section{Editora}

Centro de Investigação em Ciências Sociais

\section{Edição impressa}

Data de publição: 30 Junho 2014

Paginação: 89-102

ISBN: 1646-5075

ISSN: 1646-5075

\section{Refêrencia eletrónica}

Gloria Jólluskin, Andreia de Castro-Rodrigues, Isabel Silva e Verónica Fernandes, « Saúde e reclusão: de uma breve contextualização do fenómeno ao papel dos profissionais de saúde », Configurações [Online], 13 | 2014, posto online no dia 23 abril 2015, consultado o 02 maio 2019. URL : http:// journals.openedition.org/configuracoes/2430; DOI : 10.4000/configuracoes.2430

Este documento foi criado de forma automática no dia 2 Maio 2019

(c) CICS 


\title{
Saúde e reclusão: de uma breve contextualização do fenómeno ao papel dos profissionais de saúde
}

Health and imprisonment: from a brief contextualization of the phenomenon to the role of health professionals

Santé et emprisonnement : d'une brève contextualisation du phénomène au rôle des professionnels de santé

\author{
Gloria Jólluskin, Andreia de Castro-Rodrigues, Isabel Silva e Verónica
} Fernandes

\section{Introdução}

1 A promoção da saúde entre a população reclusa surge atualmente como uma necessidade, mas também como um grande desafio. Apesar de a população prisional ser heterogénea, em grande parte procede de sectores populacionais desfavorecidos, o que pode limitar a participação social destes indivíduos e o seu recurso aos serviços públicos, em teoria acessíveis a todos os cidadãos, como é o caso do sistema de saúde (Andrés et al., 2002; La Parra Casado, 2006).

2 Os estabelecimentos prisionais podem ser contextos promotores de saúde, tal como defende a Organização Mundial da Saúde, sendo múltiplos os exemplos de programas centrados na promoção da saúde em contexto prisional.

3 Contudo, algumas destas experiências baseiam-se numa ideia de saúde algo sofisticada, partilhada pelas classes profissionais relacionadas com a saúde, mas não necessariamente por toda a população, designadamente pelos grupos mais excluídos. Para estes, a saúde é, muitas vezes, a ausência de doença, e o seu valor é relativizado (Wilkinson \& Marmot, 2003), pelo que parece fundamental que as intervenções centradas na promoção de saúde em contextos prisionais sejam aferidas em termos de temáticas e metodologias, e avaliadas ao longo de todo o processo. 
4 Este trabalho pretende dar conta de alguns aspetos mais salientes da situação de saúde em meio prisional, refletindo sobre as inter-relações deste fenómeno com outras situações sociais e, em particular, com o papel que os profissionais de saúde podem assumir.

\section{A promoção de saúde e a reclusão}

\subsection{Alguns elementos para a caracterização da população penitenciária}

5 A planificação de uma intervenção em saúde implica a caracterização do grupo-alvo. No entanto a caracterização fi dedigna da população prisional não é uma tarefa fácil. As estatísticas oficiais costumam focar-se em variáveis como o funcionamento prisional (número total de reclusos e por tipo de estabelecimento, taxa de ocupação, entradas, saídas e transferências), as características demográficas (sexo, idade ou nacionalidade) ou os dados jurídico-legais (tipologia de crimes, penas aplicadas ou situação penal). Embora consideradas institucionalmente, as informações sobre as habilitações académicas têm uma menor presença nos relatórios anuais sobre a população prisional, sendo inexistente a referência a outro tipo de informações sobre os indivíduos, como a sua história individual e familiar (estado civil ou número de filhos), ou o contexto social de origem muito embora se entenda que esta omissão se possa fundar na tentativa de evitar a discriminação. Ainda que consideremos as estatísticas oficiais um recurso inestimável, este tipo de dados que acabámos de descrever não nos permite traçar as necessidades de intervenção, uma vez que se limita a informação relativa ao comportamento dos indivíduos, descurando a sua caracterização (cf. por exemplo, Clarke, 2013).

De modo a complementar algumas das lacunas dos dados oficiais, é frequente o recurso à investigação e à intervenção nas áreas das ciências sociais para a caracterização da população prisional. De uma forma muito global, esta população é maioritariamente constituída por homens jovens (DGRSP, 2014), frequentemente provenientes de grupos em risco social (Fariña, Arce \& Novo, 2008), para os quais a reclusão vem agravar a situação de exclusão social em que já se encontravam (Valverde, 1997).

\subsection{Exclusão e inclusão social: participação social e desigualdades sociais em saúde}

7 Neste seguimento importa operacionalizar muito brevemente o que entendemos por exclusão e inclusão. Muitas das definições destes conceitos centram-se na insuficiência de recursos como elemento central (Office for Social Inclusion, 2007: 20), aludindo-se à inclusão social como o processo através do qual os grupos excluídos conseguem participar na vida social (European Comission, 2010: 1). Neste tipo de definições assume-se a exclusão social como consequência da carência de recursos, a qual, por sua vez, dificultaria a participação social. Os dois conceitos, inclusão e exclusão social, parecem, assim, polos de um contínuo, estando determinados fundamentalmente pelos recursos materiais que os indivíduos dispõem ou não. Do nosso ponto de vista, diferentemente, a inclusão social não deve referir-se apenas à pertença ou identificação com um grupo social maioritário, com a consequente manifestação de comportamentos próprios de um determinado estilo de vida e um estatuto económico. Consideramos mais adequada uma 
conceptualização que se refira à situação em que os indivíduos iniciam processos que lhes permitem aceder de forma plena aos seus direitos e à participação social, e em que as instituições disponibilizem as respetivas e necessárias oportunidades e meios, como assinala Capucha (2005).

8 Não sendo sempre possível trabalhar diretamente os mecanismos socioeconómicos explicativos da exclusão ou as condições nas quais esta se (re)produz, entendemos ser fundamental que as intervenções no terreno de cariz social incidam sobre a capacidade de participação dos indivíduos - uma das dimensões do empoderamento. Esta é uma dimensão basilar à consecução dos objetivos de intervenção, cujo desenvolvimento poderá, por sua vez, facilitar o sucesso dos indivíduos a outros níveis. Fomentar uma maior participação dos cidadãos na vida social assume particular importância se tivermos em consideração que uma baixa participação aparece muitas vezes associada a desigualdades, injustas ou inaceitáveis, desde logo no acesso à saúde. Estas desigualdades surgem também associadas a comportamentos prejudiciais derivados da restrição dos estilos de vida, em termos da gama de opções disponíveis, acessíveis e contempláveis, ou do acesso inadequado aos serviços públicos, nomeadamente os cuidados de saúde básicos (EuroHealthNet, 2006).

\subsection{Os problemas de saúde em contexto prisional}

9 Convergindo esta discussão especificamente no contexto prisional, a literatura tem demonstrado que a situação de risco social anterior à privação de liberdade que caracteriza grande parte da população reclusa contribui para a não manutenção de um bom estado de saúde (Ramlungun, Lindsay \& Pfeif, 2001).

Efetivamente, a Organização Mundial da Saúde (2007) salienta que a população penitenciária apresenta um nível de saúde significativamente inferior à restante população, sendo que muitas pessoas que se encontram em situação de reclusão só contactam com o sistema de saúde posteriormente ao ingresso na prisão, não acedendo a programas preventivos ou assistenciais. Segundo Ferrer (2004), este défice de uso dos serviços de saúde ocorre quer por desconhecimento, quer pela não consideração da necessidade de monitorizar o estado de saúde. No geral, as situações de exclusão social têm que ver com questões específicas relacionadas com a saúde, como o desconhecimento da existência dos serviços de saúde disponíveis ou a falta de confiança na capacidade de os profissionais de saúde proporcionarem tratamentos para além dos cuidados paliativos - cenário que dificulta a procura de ajuda a nível preventivo (Ramlungun, Lindsay \& Pfeif, 2001; Howerton et al., 2007; Howerton, Byng, Campbell, Hess, Owens \& Aitken, 2007). No entanto, apesar de estes serem indicadores preocupantes, não existem muitos estudos que se centrem nas perceções e crenças que podem influenciar a procura destes serviços por parte destes grupos.

11 Por outro lado, no que respeita aos problemas de saúde em contexto prisional, várias situações revelam uma grande prevalência. Alguns estudos mostram que os reclusos costumam pedir ajuda por assuntos tão diferentes como problemas em dormir (Bjørngaard, Rustad \& Kjelsberg, 2009; Nesset, Rustad, Kjelsberg, Almvik e Bjørngaard, 2011), doenças mentais (Bjørngaard, Rustad \& Kjelsberg, 2009; Nesset et al., 2011), problemas físicos, ou abuso de drogas (Nesset et al., 2011). São igualmente frequentes, na população prisional, o VIH ou a tuberculose (Barros, Lucas \& Santos, 2010). 
12 Apesar desta multiplicidade de problemas que podem afetar a população reclusa (tomada de uma forma geral e não tendo em conta as possíveis especificidades relacionadas com distintos subgrupos: homens/mulheres; nacionais/estrangeiros), a investigação aponta para uma baixa utilização dos recursos de saúde existentes (Howerton et al., 2007). Neste sentido, Nesset et al. (2011) verificaram que apenas $66 \%$ dos reclusos utilizavam os serviços de saúde dos estabelecimentos prisionais. Segundo estes autores, não existiam diferenças no que diz respeito à utilização dos serviços, em função do sexo e/ou das habilitações académicas. Outro resultado preocupante deste estudo é que os reclusos com problemas físicos ou mentais só procuravam ajuda se existia outro problema concomitante, como, por exemplo, problemas em dormir ou consumo de drogas. Este dado parece-nos reforçar a ideia anteriormente referida de alguma falta de sensibilidade para a importância do cuidado de si próprio.

\section{O papel dos profissionais de saúde na intervenção em contexto prisional}

O Plano Nacional de Saúde 2012-2016 identifica como uma das ameaças à promoção da cidadania em saúde o "desequilíbrio de poder entre grupos sociais excluídos ou com menor empowerment, como os [...] pobres, imigrantes, reclusos" (Direção-Geral da Saúde, 2012: 7). Apesar disto, o fenómeno da reclusão, e particularmente, da saúde em contexto prisional, não tem assumido um grande destaque entre as preocupações e prioridades da classe política, em Portugal. Descurar a saúde da população prisional contudo, implica não só este grupo como aumenta a probabilidade de algumas doenças serem difundidas à população geral, quer após a libertação dos reclusos, quer através dos funcionários prisionais ou de outros profissionais que realizam as suas funções no interior da prisão, ou até dos familiares que visitam os reclusos (Comfort \& Grinstead, 2004). Esta situação pode ser agravada pela sobrelotação que caracteriza praticamente a totalidade dos estabelecimentos prisionais portugueses (DGRSP, 2014). Para além de outras questões, esta sobrelotação promove ainda a transmissão inter-reclusos de doenças infectocontagiosas, pelo facto de facilitar o desenvolvimento de comportamentos de risco e, simultaneamente, dificultar a distribuição de recursos.

É da ordem do dia que, atualmente, o país atravessa um período complicado do ponto de vista económico e financeiro, estando a acontecer cortes orçamentais mesmo em áreas fulcrais para o bem-estar da população geral. Num contexto económico tão difícil como este, não antecipamos que se realizem maiores investimentos na população penitenciária. Contudo, lembramos que a eficácia das intervenções neste contexto pode estar comprometida por questões mais psicológicas e operacionais do que financeiras. Neste sentido, sublinhamos a importância de processos elementares, fundamentais e relativamente simples e baratos de implementar, como a promoção de uma comunicação eficaz em contexto de saúde ou a promoção da literacia em saúde, processos que podem fazer diferença na potenciação dos cuidados de saúde.

\subsection{A comunicação em saúde no contexto prisional}

15 Segundo Teixeira (2004), a "comunicação em saúde diz respeito ao estudo e utilização de estratégias de comunicação para informar e para influenciar as decisões dos indivíduos e as comunidades no sentido de promoverem a sua saúde". Este autor afirma que o modo 
como ocorre a comunicação em saúde pode influenciar significativamente a adaptação psicológica à doença e os comportamentos de adesão medicamentosa e comportamental. Por outras palavras, a baixa adesão ao tratamento é identificada como uma das consequências dos problemas de comunicação na relação entre profissionais de saúde ${ }^{1} \mathrm{e}$ utentes.

Assim sendo, a comunicação em saúde globalmente considerada, bem como a qualidade da interação entre técnicos de saúde e doentes, constituem componentes essenciais de um tratamento eficaz, da qualidade dos cuidados e da segurança do doente (Bieber, Müller, Nicolai \& Hartman, 2010).

17 Os processos de comunicação exigem muitas vezes que os indivíduos sejam capazes de lidar com informação complexa, podendo incluir a avaliação da credibilidade e qualidade das informações, a análise dos riscos e benefícios de um determinado tratamento, a interpretação básica dos resultados dos exames clínicos, ou até a localização de fontes de informação ou recursos de saúde.

Assim, os indivíduos deverão apresentar capacidades informativas, sendo capazes de: obter e aplicar a informação relevante, entender o significado das probabilidades, compreender gráficos ou outras informações visuais e, em determinados casos, ter conhecimentos de informática a nível de utilizador. É também necessário ter capacidades verbais. Os utentes precisam de saber transmitir as suas preocupações com a saúde e descrever os seus sintomas com precisão, formular perguntas pertinentes e entender os profissionais da saúde ou as diferentes possibilidades de tratamento e as suas consequências.

19 Sucintamente, podemos afirmar que a comunicação em saúde neste contexto pode revelar-se especialmente difícil pelos seguintes motivos:

20 a) probabilidade de haver, entre estes indivíduos, muitas histórias de insucesso académico, que se relacionam com um vocabulário pobre, ausência de hábitos de leitura e eventuais dificuldades de aprendizagem (Zhang, Hsu, Katsiyannis, Barret \& Ju, 2011);

21 b) elevada prevalência, nestas populações, de problemas do foro emocional (Bjørngaard, Rustad \& Kjelsberg, 2009; Nesset et al., 2011);

c) percentagem de indivíduos de nacionalidade estrangeira também elevada. A DGRS (2012) indica que, no segundo semestre de 2012, existiam nas prisões portuguesas 2487 reclusos e 181 reclusas de nacionalidade não portuguesa. Contudo, muitos dos reclusos estrangeiros eram do Brasil (312 homens e 33 mulheres) ou pertenciam aos PALOP (1265 homens e 53 mulheres). Assim, poderão existir dificuldades em comunicar, ou não, em função do domínio que o indivíduo tenha da língua portuguesa, ainda que estas dificuldades possam relacionar-se com outras questões pertinentes, como as culturais e sociais, para além da língua;

d) possibilidade de existir alguma deterioração cognitiva, em alguns indivíduos, resultante do consumo de drogas ilícitas.

Esta realidade decorre frequentemente num quadro geral de falta de compreensão das mensagens, comprometedora de uma comunicação eficiente, dificuldades que ainda se ampliam mais em circunstâncias stressantes como pode ser o contexto clínico. Crawford e Bull (2006) encontraram, em jovens que tinham cometido uma infração, dificuldades de organização narrativa da informação de forma lógica e sequencial, capacidades fundamentais tanto nos processos judiciais como nas intervenções clínicas. Estas 
dificuldades podem também torná-los mais vulneráveis, uma vez que o seu comportamento pode ser interpretado pelos profissionais como uma manifestação de tédio, um comportamento evasivo ou falta de vontade de cooperar. Além disto, os indivíduos, por sua vez, podem tentar dissimular os eventuais problemas de fala, linguagem e/ou comunicação, evitando o envolvimento na situação ou apresentando comportamentos disruptivos com o objetivo de desviar a atenção daqueles problemas (Humber \& Snow, 2011).

Apesar de uma boa comunicação e uma boa relação entre profissionais de saúde e utente estarem associadas a claros ganhos, a investigação sugere que a maioria dos técnicos não se sente confiante nas suas competências de comunicação (Servellen, 2009). Mesmo quando doentes e profissionais demonstram estar satisfeitos com a comunicação existente entre eles, é possível que esta não seja completamente eficaz (Roscoe, 2013). No contexto prisional, a comunicação em saúde parece constituir-se como um desafio ainda mais difícil para os profissionais de saúde do que seria noutros contextos, mas, do nosso ponto de vista, será fundamental para o sucesso do tratamento prisional.

\subsection{A literacia em saúde no contexto prisional}

Importa fazer um recuo para definir que entendemos por literacia em saúde. A literacia em saúde é definida como "o grau no qual os indivíduos são capazes de obter, processar e compreender informações para tomar decisões de saúde básicas adequadas e utilizar os serviços necessários" (Nielsen-Bohlman, Panzer \& Kindig, 2004). Estão portanto implicados processos de análise da informação e de tomada de decisão, bem como a capacidade de aplicar as decisões à situação. A literatura mostra que indivíduos com um baixo nível de literacia em saúde apresentam um estado de saúde mais deficitário e uma menor probabilidade de utilizar métodos preventivos em relação aos seus problemas ( ibidem, 2004). Os estudos também identificam alguns grupos que apresentam uma maior fragilidade, como as minorias étnicas, pessoas com experiência de doenças físicas ou mentais crónicas, ou pessoas de baixos recursos económicos (National Assessment of Adult Literacy, 2006). Algumas destas características são frequentes entre a população penitenciária.

Nesta sequência, defendemos a importância de aumentar a literacia em saúde entre os indivíduos que cumprem uma pena privativa de liberdade no sentido de assegurar o sucesso dos programas em funcionamento e melhorar a adaptação à situação de reclusão, mas também para garantir o bem-estar da população geral.

\section{Implicações para a prática}

Dado o papel que os profissionais de saúde desempenham no cuidado de pessoas em situação de reclusão, concordamos com Howerton et al. (2007) quando afirmam que é fundamental que estes profissionais recebam um treino adequado para conseguir reduzir de forma efetiva os problemas de saúde nesta população.

Segundo estes autores, os profissionais de saúde não costumam estar familiarizados com as especificidades do contexto e da população reclusa, recebendo essencialmente formação para trabalhar com "populações normativas", sinalizadas e tratadas em contextos menos restritivos. Por outro lado, quem trabalha no contexto prisional nem 
sempre conta com os mesmos recursos humanos e materiais, em particular a nível preventivo, que existem no meio livre (Ferrer, 2004). Além disso, durante a sua formação, os profissionais são incentivados a fomentar a autonomia no utente dos serviços de saúde, o que pode ser particularmente desafiante quando o indivíduo está privado de liberdade (Birmingham, Wilson \& Adshead, 2006).

Neste sentido da facilitação da comunicação entre profissionais e reclusos, encontram-se na literatura muitas propostas de medidas e iniciativas. Uma das que consideramos particularmente completas e simultaneamente passíveis de transpor para diferentes realidades como Portugal é a da organização Nacro (2011), que sistematiza uma série de recomendações concebidas especificamente para o trabalho clínico com ofensores, das quais destacamos:

31 1. dar tempo ao indivíduo para ouvir e entender o que está a ser dito, enfatizando as palavras em que a atenção se deve centrar e utilizando frases curtas e pausas que permitam o processamento de novas informações;

2. perguntar se é possível fazer algo para ajudar a compreender melhor o que está a ser dito;

33 3. solicitar ao indivíduo que repita com as suas próprias palavras a informação que foi transmitida;

34 4. verificar se o indivíduo compreendeu o que se espera dele;

35 5. lembretes de todos os passos a dar em relação ao tratamento ou dos compromissos estabelecidos;

6. esclarecer tudo aquilo que o indivíduo diga e que o profissional não perceba;

7. começar a sessão por uma versão resumida das questões a abordar antes de passar a uma explicação mais aprofundada;

8. proporcionar-lhe reforço positivo e feedback.

9. simplificar os materiais escritos utilizados, optando por uma linguagem simples, uma escrita clara e uma terminologia compreensível; criar materiais em diferentes línguas ou adaptados a diferentes grupos culturais;

10. recorrer a ferramentas visuais para incentivar a sua compreensão.

41 Os profissionais da saúde assumem, efetivamente, um papel importante na promoção do desenvolvimento da literacia de saúde, quer seja através do fornecimento de materiais, quer desenvolvendo ativamente programas de alfabetização de saúde, por meio de parcerias com organizações de base comunitária, centros de ensino, associações dedicadas à alfabetização de adultos, ou associações de utentes.

Como temos vindo a argumentar, este objetivo de melhorar a alfabetização em saúde e as competências sociais, parece essencial no contexto prisional, no sentido de favorecer a adesão ao tratamento, assim como contribuir para a redução da reincidência através do desenvolvimento de competências, da promoção do empoderamento e da responsabilização dos indivíduos pelo seu estado de saúde (Vigilante et al., 1999). Consideramos ainda que desenvolver o hábito de comunicar eficazmente em saúde ajudaria a desenvolver importantes competências nos indivíduos, mesmo quando estes apresentem características que tornem a comunicação difícil.

Para finalizar esta epígrafe, gostaríamos de insistir na necessidade de ir além da intervenção individual, nomeadamente desenvolvendo intervenções que permitam 
reduzir as diferenças sociais. Com o objetivo de guiar o trabalho do profissional no terreno, salientamos a proposta do Governo australiano (2010) de boas práticas de estratégias de inclusão social, que tentámos adaptar à realidade portuguesa. A primeira destas propostas consiste em aproveitar os pontos fortes individuais e comunitários, atendendo particularmente aos grupos culturais ou étnicos minoritários. A segunda passa pelo estabelecimento de parcerias com as principais partes interessadas (Estado, organizações e comunidades, etc.), no sentido do trabalho conjunto para obter os melhores resultados para as pessoas em necessidade. Por outro lado, salienta-se também a importância de desenvolver serviços adaptados com o objetivo de trabalhar conjuntamente para identificar formas novas e flexíveis de atender às diferentes necessidades de cada pessoa e grupo social. Outra sugestão vai no sentido de priorizar a prevenção e intervenção precoce, centrando a atenção na compreensão dos problemas e populações e realizando intervenções antecipando as consequências previsíveis.

Uma outra sugestão em ligação com esta diz respeito à importância de utilizar as evidências científicas para traçar políticas de intervenção. O sucesso das intervenções reside, em parte, na descoberta dos princípios subjacentes à aplicação das mesmas, pelo que parece fundamental identificar as estratégias que mostraram ter sucesso e divulgar os procedimentos e resultados dos programas aplicados, refletindo de forma crítica sobre os mesmos (inclusivamente, ou até com mais motivo, quando os resultados foram negativos). Outro ponto referido naquele documento é o apelo à utilização de abordagens locais, trabalhando em contextos sociais desfavorecidos, com o objetivo de chegar às pessoas que têm mais necessidades de intervenção, tentando compreender como os diferentes problemas da comunidade se relacionam. Por fim, aconselha-se a planificação da sustentabilidade da intervenção, fortalecendo ao mesmo tempo as pessoas para que consigam lidar com os seus problemas futuros, e não unicamente com aquela situação que tentam resolver através da intervenção.

\section{Reflexão final}

A crise do sistema penitenciário não se refere apenas à relativa eficácia que a pena privativa de liberdade, tal como hoje é aplicada, tem demonstrado (Gonçalves, 2009), mas também ao abandono e esquecimento da população reclusa por parte da sociedade em geral, e da classe política em particular. Este esquecimento assenta, desde logo, na consideração falaciosa das problemáticas associadas à prisão e as suas múltiplas implicações como um problema periférico e irrelevante.

A população penitenciária constitui efetivamente um grande coletivo. Em maio de 2014 existiam 14299 reclusos nas prisões portuguesas (DGRSP, 2014).

Esta cifra constitui um aumento significativo em relação às estatísticas anteriores, que situavam a população prisional, a 1 de novembro de 2012, em 13630 indivíduos (DGRS, 2012). Não devemos esquecer que os problemas de saúde desta população (e de qualquer outra) devem ser considerados de forma sistémica, isto é, envolvendo a família, e que devemos igualmente considerar os profissionais que desenvolvem as suas funções nos estabelecimentos prisionais (e por sua vez, as respetivas famílias). Corroboramos portanto, a ideia de que os problemas de saúde em contexto prisional são uma questão de saúde pública (United Nations Office on Drugs and Crime, 2013). 

a Lei n.. 115/2009, de 12 de outubro, no artigo 7.ํ, garante que o recluso tem o direito a "ter acesso ao Serviço Nacional de Saúde em condições idênticas às que são asseguradas a todos os cidadãos". Porém, do nosso ponto de vista, a sociedade não compreende os problemas de saúde (e outros) que apresenta a população prisional, nem está consciente de como estes problemas podem afetar toda a comunidade. Esta situação pode resultar em reações de incompreensão perante a defesa da aplicação do princípio de equivalência assistencial, recomendado pelas Nações Unidas, isto é, que os serviços recebidos pelos reclusos sejam da mesma qualidade daqueles que o Estado disponibiliza para a população geral. Mais concretamente, nos Princípios Básicos Relativos ao Tratamento de Reclusos Adotados e proclamados pela Assembleia Geral das Nações Unidas na sua resolução n.. 45/111, de 14 de dezembro de 1990, refere-se que "os reclusos devem ter acesso aos serviços de saúde existentes no país, sem discriminação nenhuma decorrente do seu estatuto jurídico".

Pelo exposto, consideramos fundamental a implementação de políticas públicas relacionadas com a saúde da população penitenciária. Contudo, pensamos que é necessário que essas políticas excedam este nível de atuação, centrando-se no problema desde a sua origem, através da aplicação de programas de prevenção primária da criminalidade eficazes. Isto facilitaria a diminuição das diferenças sociais, minimizando consequentemente o surgimento de problemas sociais e de saúde. No entanto, a defesa da ênfase no nível primário da prevenção não descura a pertinência da intervenção ao nível da prevenção secundária e terciária. Assim, seria sempre necessário desenvolver políticas e implementar soluções relativamente aos problemas e necessidades da população prisional. Em qualquer dos casos, as medidas aplicadas deveriam ser sempre críticas quanto à possibilidade de estarmos a perpetuar um ciclo em que a justiça criminaliza a escassez de recursos dos indivíduos.

53 Terminamos com o apontamento de Bellver (2006) relativamente à necessidade de os profissionais de saúde que trabalham no terreno manterem o seu empenho profissional num ambiente tão difícil como o prisional. Pensamos que isto depende da adequada 
formação dos profissionais, não só do ponto de vista técnico, como também do ponto de vista ético-moral, nomeadamente no que respeita a uma atuação desprovida de preconceitos. Tal como Bellver afirma: "Os profissionais da saúde dedicados a estes contextos têm a dupla responsabilidade de exigir à sociedade o cumprimento destes deveres e de esforçar-se por atingir a excelência ética nestas funções, muito embora frequentemente careçam dos meios necessários e do enorme reconhecimento social que lhes corresponderia" (Bellver, 2006: 9).

\section{BIBLIOGRAFIA}

ANDRÉS, Josep de; BARRENTI, Mila; BORRELL, Carme; CALAFELL, Joana; PASARIN, Maria Isabel; PUIGPINOS, Roser \& JANSÁ, Josep (2002), "Salud y marginación social”, Documentación social: Revista de Estudios Sociales y de Sociología Aplicada, volume (127): 97-123.

AUSTRALIAN GOVERNMENT (2010), Social Inclusion Principles for Australia. [Online], disponível em: http://www.meetingpoint.org.au/assets/mp_s12_sipfa.pdf [consultado em: 27 de abril de 2014].

BARROS, Henrique de; LUCAS, Raquel \& SANTOS, Maria José (2010), Sida em meio prisional. Lisboa: Fundação Calouste Gulbenkian.

BELLVER CAPELLA, Vicente (2006), “Ética, salud y atención sanitaria en las prisiones”, Revista Española de Sanidad Penitenciaria, volume 9: 7-9.

BIEBER, Christiane; MÜLLER, Knut; NICOLAI, Jennifer; HARTMANN, Metchhild \& EICH, Wolfgang (2010), "How does your doctor talk with you? Preliminary validation of a Brief Patient Self-Report Questionnaire on the Quality of Physician-Patient Interaction", Journal of Clinical Psychology in Medical Settings, volume 17 (2): 125-136.

BIRMINGHAM, Luke; WILSON, Simon \& ADSHEAD, Gwen (2006), "Prison medicine: ethics and equivalence", The British Journal of Psychiatry, volume 188: 4-6.

BJøRNGAARD, Johan Håkon; RUSTAD, Åse-Bente \& KJELSBERG, Ellen (2009), “The prisoner as patient - A health service satisfaction survey", BMC Health Services Research, volume 9: 176-184.

CAPUCHA, Luís (2005), Desafios da pobreza. Oeiras: Celta Editora.

CLARKE, Steven (2013), Trends in Crime and Criminal Justice, 2010 [Online], disponível em: http:// epp.eurostat.ec.europa.eu/cache/ITY_OFFPUB/KS-SF-13-018/EN/KS-SF-13-018-EN. PDF [consultado em: 30 de julho de 2014].

COMFORT, Megan L. \& GRINSTEAD, Olga (2004), "The carceral limb of the public body: Jail inmates, prisoners, and infectious disease", Journal of the International Association of Physicians in AIDS Care, 3 (2), 45-48.

CRAWFORD, Emma \& BULL, Ray (2006), “Teenagers' diffi culties with key words regarding the criminal court process", Psychology, Crime \& Law, volume 12 (6), 653-667.

DIREÇÃO-GERAL DA SAÚDE (2012), Plano Nacional de Saúde 2012-2016. 3.1 Eixo Estratégico - Cidadania em saúde [Online], disponível em http://pns.dgs.pt/fi

les/2012/02/99_3_1_Cidadania_em_Saúde_2013_01_17_.pdf [consultado em: 3 de julho de 2014] 
DIREÇÃO-GERAL DOS SERVIÇOS PRISIONAIS (2012), Estatísticas Prisionais. 2.ํㅗ semestre de 2012. [Online], disponível em: http://www.dgsp.mj.pt/backoffi ce/Documentos/DocumentosSite/ Estatisticas_e_Relatorios/2-trimestre-2012.pdf [consultado em: 15 de abril de 2014].

DIREÇÃO-GERAL DE REINSERÇÃO E DOS SERVIÇOS PRISIONAIS (2014), População prisional, por tipo de estabelecimento, segundo a situação penal em 15 de junho e 1 de julho de 2014. [Online], disponível em: http://www.dgsp.mj.pt/backoffice/uploads/ quinzenais/20140701030754SitPen_15jun-1jul.pdf [consultado em: 15 de abril de 2014].

EUROHEALTHNET (2006). Annual Report 2006. [Online], disponível em: http://eurohealthnet.eu/ sites/eurohealthnet.eu/fi les/publications/EHN_ANNREP_2006.pdf [consultado em: 18 de janeiro de 2014].

EUROPEAN COMISSION (2010), The European Social Fund and Social Inclusion [Online], disponível em: http://ec.europa.eu/employment_social/esf/docs/sf_social_inclusion_en.pdf [consultado em: 15 de novembro de 2013].

FARIÑA, Francisca; ARCE, Ramón \& NOVO, Mercedes (2008), “Neighborhood and community factors: Effects on deviant behavior and social competence", The Spanish Journal of Psychology, Volume 11 (01), 78-84.

FERRER, Ventura (2004), “Talleres de Educación para la Salud”. Revista Española de Sanidad Penitenciaria, volume 6, 15-19.

HOWERTON, Amanda; BYNG, Richard; CAMPBELL, John; HESS, David; OWENS, Christabel \& AITKEN, Peter (2007). "Understanding help seeking behaviour among male offenders: qualitative interview study”, British Medicine Journal [Online], disponível em: http://www.antoniocasella.eu/ archipsy/Howerton_2007.pdf [consultado em: 15 de outubro de 2014].

HUMBER, E. \& SNOW, P. (2001), “The oral language skills of young offenders: a pilot investigation", Psychiatry, Psychology and Law, volume 8, 1-11.

LA PARRA CASADO, Daniel (2006), Hacia la equidad en salud. Estudio comparativo de las encuestas nacionales de salud a población gitana y población general de España, 2006. [Online], disponível em: http://ec.europa.eu/health/social_determinants/docs/spain_rd03_en.pdf [consultado em: 1 de agosto de 2014].

NACRO (2011), Speech, Language and Communication Diffi culties. Young people in trouble with the law [Online], disponível em: http://www.nacro.org.uk/data/fi les/speech-languagecommunications-954.pdf [consultado em: 20 de novembro de 2013].

NATIONAL ASSESSMENT OF ADULT LITERACY (2006), The Health Literacy of America's Adults. Results from the 2003 National Assessment of Adult Literacy. [Online], disponível em: http://nces.ed.gov/ pubsearch/pubsinfo.asp?pubid=2006483[consultado em: 20 de novembro de 2013].

NESSET, Merete Berg; RUSTAD, Åse-Bente; KJELSBERG, Ellen; ALMVIK, Roger \&

BJøRNGAARRD, Johan Håkon (2011), "Health care help seeking behaviour among prisoners in Norway", BMC Health Services Research, 11, 301.

NIELSEN-BOHLMAN, Lynn; PANZER, Allison M. \& KINDIG, David A. (Eds.) (2004), Health Literacy: A prescription to end confusion. Washington, D.C.: The National Academies Press.

OFFICE FOR SOCIAL INCLUSION (2007), National Action Plan for Social Inclusion 2007-2016. [Online], disponível em: http://www.socialinclusion.ie/documents/NAPinclusionReportPDF.pdf [consultado em: 2 de junho de 2014]. 
RAMLUNGUN, Pras; LINDSAY, Bruce \& PFEIF, Michael (2001), “Research in prison: a researcherpractitioner's view", Nurse Researcher, 17 (3), 60-71.

ROSCOE, Lori; TULLIS, Jillian; REICH, Richard; McCAFFREY, Judith (2013), “Beyond good intentions and patient perceptions: Competing definitions of effective communication in head and neck cancer care at the end of life", Health Communication, volume 28 (2): 1-10.

SERVELLEN, Gwen van (2009), Communication Skills for the Health Care Professional. Concepts, practice and evidence. USA: Jones and Bartlett Publishers.

TEIXEIRA, José A. (2004), “Comunicação em saúde. Relação técnicos de saúde-utente”, Análise Psicológica, volume 22(3): 615-620.

UNITED NATIONS OFFICE ON DRUGS AND CRIME (2013), “Good governance for prison health in the 21st century”. [Online], disponível em: http://www.unodc.org/documents/hiv-aids/ publications/Prisons_and_other_closed_settings/Good-governance-for-prison-healthinthe-21st-century.pdf [consultado em: 25 de julho de 2014].

VALVERDE, Jesús (1997). La cárcel y sus consecuencias, La intervención sobre la conducta desadaptada. Madrid: Editorial Popular.

VIGILANTE, Kevin C.; FLYNN, Mary M.; AFFLECK, Patricia C.; STUNKLE, Julia C.;

MERRIMAN, Nathan A.; FLANIGAN, Timothy P.; MITTY, Jennifer A. \& RICH, Josiah D. (1999), "Reduction in recidivism of incarcerated women through primary care, peer counseling, and discharge planning", Journal of Women's Health, 8(3): 409-415.

WILKINSON, Richard \& MARMOT, Michael (2003), Los determinantes sociales de la salud, Los hechos probados. Madrid: Ministerio de Sanidad y Consumo.

ZHANG, Dalun; HSU, Hsien-Yuan; KATSIYANNIS, Antonis; BARRETT, David \& JU, Song (2011), "Adolescents with disabilities in the juvenile justice system: Patterns of recidivism", Exceptional Children, 77: 283-298.

\section{NOTAS}

1. Antes de mais importa operacionalizar que, quando falamos em profissionais de saúde neste contexto prisional, referimo-nos em concreto aos vários protagonistas dos cuidados de saúde aos reclusos, ou seja, médicos/as, médicos/as dentistas, enfermeiros/as, psicólogos/as, etc.

\section{RESUMOS}

Os grupos em situação de exclusão social estão sobrerrepresentados na população reclusa, sendo que as desigualdades económicas e sociais estão muitas vezes relacionadas com diferenças no acesso aos serviços de saúde. Como resultado, a população reclusa apresenta um nível de saúde significativamente inferior ao da população geral. Os profissionais de saúde que trabalham em contextos prisionais confrontam-se, assim, com uma série de dificuldades, ligadas não só a aspetos próprios do fenómeno da reclusão, como também a particularidades da própria 
população. No presente trabalho partimos da reflexão em torno de algumas questões relacionadas com a saúde em contexto prisional, chegando a um conjunto de medidas e princípios operativos.

Prison population is overrepresented by social disadvantaged groups. These economic and social inequalities are often related to differences in access to health services. As a result, prison population has a lower level of health compared to general population. Health care providers must face a number of difficulties, which are not only linked to the characteristics of confinement, but also derived by individual characteristics. In this work, we start from a reflection on some issues related to health in the prison context, arriving at a set of measures and operating principles.

Les groupes en situation d'exclusion sociale sont surreprésentés dans la population carcérale. Ces inégalités économiques et sociales sont souvent liées à des différences dans l'accès aux services de santé. En conséquence, la population carcérale présente un niveau de santé nettement inférieur à celui de la population en général. Le professionnel de santé travaillant en milieu carcéral est confronté avec un certain nombre de difficultés, liées non seulement aux caractéristiques du phénomène d'emprisonnement, mais également à des spécificités provenant de la population elle-même. Avec ce travail nous nous appuyons sur une réflexion autour de certaines questions liées à la santé en milieu carcéral, afin d'arriver à une série de démarches et principes opérationnels.

\section{ÍNDICE}

Mots-clés: Population carcérale, promotion de la santé, communication et santé, éducation en matière de santé

Palavras-chave: População reclusa, promoção da saúde, comunicação em saúde, literacia em saúde

Keywords: Prison population, health promotion, health communication, health literacy

\section{AUTORES}

\section{GLORIA JÓLLUSKIN}

Universidade Fernando Pessoa, Faculdade de Ciências Humanas e Sociais. Endereço de correspondência: Praça 9 de Abril 349, 4249-004 Porto, Portugal

gloria@ufp.edu.pt

\section{ANDREIA DE CASTRO-RODRIGUES}

Universidade Fernando Pessoa, Faculdade de Ciências Humanas e Sociais. Endereço de correspondência: Praça 9 de Abril 349, 4249-004 Porto, Portugal

andreiagtcr@gmail.com

\section{ISABEL SILVA}

Faculdade de Ciências Humanas e Sociais da Universidade Fernando Pessoa (UFP). Endereço de correspondência: Praça 9 de Abril 349, 4249-004 Porto, Portugal isabels@ufp.edu.pt 


\section{VERÓNICA FERNANDES}

Universidade Fernando Pessoa, Faculdade de Ciências Humanas e Sociais. Endereço de correspondência: Praça 9 de Abril 349, 4249-004 Porto, Portugal

15256@ufp.edu.pt 